Dermatologische Zeitschrift. 1899;6:I-VI

\title{
Contents, Vol. 6, 1899
}

Dermatologisehe Zeitsehrift.

Herausgegeben

Prof. Dr. O. LASSAR.

Band VI. 1899.

Mit 4 Tafeln und zahlreichen Abbildungen im Text.

$\& \uparrow \mathrm{f} \kappa$.

Berlin 1899.

VERLAG VON S. KARGER

KARLSTRASSE 15.

Alle Rechte vorbelialten.

Gedruckt bei Imberg \& Lefso $\alpha$ in Berlin SvV.

Inhalts-Verzeichnis.

HEFT i.

Seite

Experimentelle und klinische üntersuchu ugen

iiber die reducierenden Wírkungen des

Pyrogallo Is, Eugallols und Lenigallols. Von

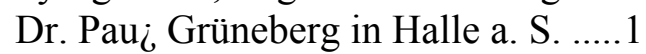

Ueber Lepra-Bekandlun g mit merk uri elleu In-

jectionei). Von Prof. Dr. Alex Hasíund in Kopen-

hagen 29

Zur L·ar gin-Therapie der Gonorrhoea muliebris.

Von San.-Rat Dr. L. Fürst in Berlin 38

Teber deuWert des Oleum Salosantali (Salo-aantal) für die interne Behaiidlung der Harnkrank-

heiten. Von Dr. Leop. Halm in Berlin . . 4 4(5

Indurati onen i $\mathrm{m}$ Corpus cavernosum penis. Von

Dr. Echtermeyer in Berlin. (Hierzu 4 Abbildimgen) 68

Zur Pathogeuese der localisirten Antipyrinex-

antheme. VГon Dr. Hugo Apolaiit in Berlin . . 67

Die Dermatolo gischen Compl ication en des

Diabetes und ihre Therapie. Von O. Lassar 71

Berliner Dermatologisch e Gesell»eha ft.

Sitzuhg yom G. Dezember 1898 79

Franz ösisch'e Gesellschaft für Dermatologie and Syphiligraphie.

Sitzung vom 10. November 1898 . . . . 92

8. Dezember IS98 93

Periodíscne Litteratur 94

Besprechungen .......... 123 
Fiestschrift, gewidmet Filipp Josef Pick, aus Anlass der Vollendung seiner 2õjährigen Thätigkeit als Professor und Vorstand der K. K. dermatolo gischen Klinik in Prag . . . 1ST

B u chanzeige n . . . 145

Berichtigung. $\quad 151$

Practische Notizen 152

Person alie $\mathrm{n}$. Tagesnach ricbteu $\mathrm{u}$ nd Notizen .152

HEFT 2..

Ueber eine besondere Form von Acne arteficialis.

Von Prof. Dr. Rilíe in Innsbruck. (Hierzu Tafel I) 153 Ueber die VerwentdtiTig von Aethal (Cetylalkohol)

in der Hautpflege. Von Dr. F Grimm in Berlin 158 Zur Geschiehte der Lepra. Von Dr. Gemy in Akier 160

$-\mathrm{IV}$

XI. Ueber Ichthyosis. Von Dr. Max Penkert . . . 171 XII. Der elektrische Mikroh eissluftkauter. Von

Dr. E. Hollander in Berlin. (Mit 2 Abbildungen) 204 Berliner dermatologische Gesellschaft.

Sitzung voni 10. Januar 1899 , 206

Französische Gesellschaft für Dermatologie und Syphilographie.

Sitzung rom 12. Januar 1899223

Moskauer Dermatologische und Venereolo-gischen Gesellschaft.

Sitzung vom 4. Dezember 1898 . . . . 225

,18. „, $1898 \quad 226$

„22. Januar 1899 ..... 228

5. Februar $1899 \quad 229$

„31. März $1899 \quad 231$

Dänische dermatologische Gesellschaft in K op en hag en.

I. Sitzung vom 4. Januar 1899233

II. , „1. Februar $1899 \quad 239$

III. , , , 1. März $1899 \quad 245$

Niederländische dermatologische Gesellschaft.

Bericht über das Jahr 1897/98*253

Italienische Gesellschaft für Dermatologie und Sifiligraphie.

Jahres-Sitzung Dezember $1897 \quad 256$

Periodiscbe Litteratur 261

Besprechungen 284

Buchanzeigen 285

Dr. Unna's Dermatologische Preisaufgabe . . 287

Tagesnachrichten 288

HEFT 3.

XIII. Eine neue Lupusbehandlung durch innere Medi-

cation. Von Dr. A. Philippson in Hamburg . .289

XIV. DieWirkungen derLippspringer,_Arminiusquelle” 
auf Haut-Erkrankuagen (speciell Lupus exul-cerans). Von Dr. Rohden in L·ippspringe . . 294 XV. Erythromelalgie und Hautatrophie. Von Dr.

7?. Schütz in Wiesbaden 297

XVI. Das lösliche metallíscbe Quecksilber als Heil-mittel. Klinisch-therapeutische

Untersuchunge $3 / 4$ yon Dr. med. Oscar Werler in Berlin . . . . . 303

XVII. Fremdkörper der Hamröhre. Von Dr. M. Fried-

länder 333

Bericht über die im Dermatologischen Central-

blatt erschienenen Ori ginalarb eiten .... 335 Berliner Dermatologische Gesellschaft.

Sitzung rom 7. Februar 1899346

„7. März $1899 \quad 361$

-21. , 1899377

- VI -

Kranklieiten. Von Dr. mod. Fr. E,.Hópf in

Dresden no 7

Beriфht iiber die dermatologist; he Section der Naturforscher-Versammluiig von Dr. P,Meissner iu Berlin '. 645

Berliner Derniatologisehe Gesellschaft.

Sitzung vom 6. Juni 1899

659

,. 4. Juli 1899675

B u chauzeigen $\quad 693$

Tagesnachrichteii 694

Practische Notiz $\beta$ n $\quad 694$

$\operatorname{HEFT}(\mathrm{k}$

XXVII ZweiFäile von Alopecie nacli dern Gebrauc li von

Thalliumacetat. Von Prof. $>$ \$. Giovannini in Turin 695 XXVIII. Bericlit über die Lepra in NeuSüd-Wjales für "il

das Jahr 1897. Von J. Ashburton Tompson . 704 XXIX. Talgdrüsen in der Sehleimhaut des

Mundes. Von ' $\bullet$ '..

Prof. Douglas, W. Montgommery und W. S. Hay 716 XXX. Ein Fall von Pemphigus “ folliaceus

Ca-zenavei..;,

Von Dr. G M. Nasarozv in Odessa 719

XXXI. Zur Geschichte der Lepra in den Niederläirdisch-ostindischen Kolonien $\tau$ om 17.

Jahrhundert bis jetzt. Von T. Broes van Dort in :Roíterdam 728 Dänische dermatologische

Gesellschaft. ;: ; Xк'k

Sitzong am 3. Mai $1899 \ldots \wedge 1 \ldots 750$

Sitzung am 4. October 1899 . . ;.L . . 759 7. Pirogow’scher Congress russisch·er Äei”’3/4te zu ïvasan. Section für Dernxato-Venerotógie.

Sitzung “vom 19. April 189.9 ., . .'íivs.' . 770 „, 30. April: 18 9ftv:.í : . . . 770

3. Mai 1899 J ...... 771

Sitzung der vereinigten Sectionen für Dermato-Venerologie und Infectionskrankheiten am

3. Mai ....... 773

Berliner Dermatologische Gesellschaft.

Sitzung vom 7. November $1899 \quad 774$

Periodische Litteratur

Bespre.chungen '..', 
Buchanzeige :

Tagesnachrichten und Notizen . . . .... X

Die Verbreitung der Syphilis, der venerischen

Krankheiten und der Prostitution in Russland

von Prof. Dr. O. v. Petersen und Dr. C. v. Stunner

in St. Petersburg. $\quad$ Ergänzungsheft

$\mathrm{V}-$

American Dermatological Association.

Sitzung vom 31. Mai uud 1. Juni 1898 in Princeton und vom 2. Juni 1898 in

New-York City ."....... 391

Periodische Litteratur $\quad 399$

Besprechungen 422

Buchanzeigen $\quad 428$

Practische Notizen 431

Örthoform . 431

Litterarisch e Notizen . . . . . . . . .

Tagesnachrichten

431

Das neue Lepraheint

431

Personal! en und Notizen

$$
432
$$

HEFT 4.

XVIII Die Diagnose und ßehandlung der Gon $\pi$ orrhoe

beim Wei be. Von Dr. Adolf Ca¿mann in Hamburg 433 XIX. Zur Pemphigusfrage. Von Dr.

Friedrich Luithíen

in $\Lambda$ Vien $\quad 481$

XX. Ueber einen Fall von Lepra tuberosa ohne Be-fund von Leprabacillen und über das Vor. kommen von Riesenzellen in leprösen Haut-infiltraten. Von Dr. Carl Bvutzer in Riga. (Hierzu Tafel IV) 491

XXI. Ueber Auftreten von universellem Exanthem nach localer Anwendung von Jodvasogen.

Von

Dr. L. Lipman-Wulf vá Berlin 499

Berliner Dermatologische Gesellschaft.

Sitzung vom 2. Mai 1899 ....... 502 Dänische dermatologische Gesellschaft.

4. Sitzung vom 5. April 1899521

Periodische Litteratur 533

Besprechungen .

565

Buchanzeigen 574

Tagesnachrichten und Notizen

575

HEFT 5.

XXII. Beitrag zur Diagnostik der Hautgummen. Von

Dr, Arnold Loewald 577

XXIII. Beitrag zur ßehandlung der Akne vulgaris. Von

Dr Jul. Müller in Wiesbaden 583

XXIV. Das Aussätzigen-Asyl „Jesus-Hilfe” bei Jerusalem

und der Aussatz in Palästina. Von Geh. Ober.-Med.-Rat Dr. Schmidtmann in Berlin . . . . 588

XXV. Uebei die Verbreitung der venerischen Krank- 
heiten. Von Prof. Dr. O. Lassar in Berlin . 596 XXVI. Bericht über die internat, Konferenz zur Pro-phylaxc der Syphilis und der venerischen 